\title{
Fine-tuning type I IFN signaling: A new chapter in the IFN
}

\section{saga}

Cell Research (2017) 27:1407-1408. doi:10.1038/cr.2017.118; published online 19 September 2017

Type I interferon (IFN) signaling is critical for intracellular antimicrobial programmes, affecting both innate and adaptive immune responses. The paper recently published in Cell demonstrates a new regulatory mechanism of the type I IFN signaling pathway by histone-lysine N-methyltransferase SETD2.

The Janus tyrosine kinase (JAK), Signal Transducer and Activator of Transcription (STAT) proteins, and Interferon Regulatory Factor (IRF) family of transcription factors are important mediators of many cytokine systems [1-4]. While cytokine research has impacted a number of fields, it is remarkable that the major cytokine pathways were first elucidated in the context of the same regulatory network, the type I interferon (IFN) system. As such, one may term the series of studies as a first wave of research on cytokine induction and action.

The field may now be witnessing a so-called second wave of discovery related to how cytokine signaling systems are fine-tuned by other regulatory pathways. This is exemplified by a recent paper by Cao laboratory who demonstrate that type I IFN-induced STAT1 activation is regulated by histone-lysine N-methyltransferase SETD2 (SET domain containing 2), which has been previously characterized for its trimethylation of lysine 36 of histone H3 (H3K36) [5]. The authors first performed high-throughput screening of epigenetic modifiers by lentivirusmediated RNAi (RNA interference) to search for epigenetic factors that modu- late type I IFN-mediated inhibition of HBV (hepatitis B virus) replication in HBV-transfected hepatocyte cell line HepG2.2.15. This screen allowed them to identify 37 candidate genes. The authors then proceeded to validate each candidate gene individually by small interfering RNA (siRNA) mediated gene silencing, which revealed that suppression of SETD2 showed a marked elevation of HBsAg (HBV surface antigen) secretion and HBV DNA in response to IFN- $\alpha$ stimulation. Remarkably, the importance of SETD2 in the IFN-mediated suppression of HBV replication was further evaluated by generating $\operatorname{Setd} 2$ conditional knockout (KO) mice with hepatocyte-specific deletion of Setd2. In these mice, they observed significantly higher HBsAg titers and severe liver injury as compared to control mice, clearly demonstrating a critical role of SETD2 in type I IFN-mediated inhibition of HBV replication.

How does SETD2 enhance type I IFN-mediated antiviral activity? Chen et $a l$. [5] found that IFN- $\alpha$-induced STAT1 phosphorylation, STAT1 nuclear localization and induction of a STAT1 target gene, ISG15 (IFN-stimulated gene 15), were all diminished in SETD2-deficient HepG2 cell lines (SETD2-KO cells) and primary hepatocytes from Setd 2 conditional KO mice. Impaired STAT1 activation was also observed when SETD2-KO cells were stimulated by other types of IFN, namely, IFN- $\gamma$ and IFN- $\lambda$, indicating there is a shared SETD2-mediated regulation of STAT1 downstream of the three distinct classes of IFN receptors [6]. In contrast, over- expression of the SETD2 SET domain (SETD2-F2) in SETD2-KO cells restored IFN- $\alpha$-induced ISG15 mRNA expression, indicating the critical role of the SET domain.

In further addressing the mechanism underlying SETD2-mediated enhancement of STAT1 activation, Chen et al. interestingly found that STAT1 serves as a target of mono-methylation upon IFN- $\alpha$ stimulation. Mass spectrometry analysis revealed seven monomethylated lysine residues in STAT1. Among them, the authors identified mono-methylation on $\mathrm{K} 525$ residue (K525me1) of STAT1 to be critical for IFN- $\alpha$-induced STAT1 interaction with JAK1 kinase and for phosphorylation, nuclear translocation, and DNA-binding activity of STAT1. Since SETD2 is a known histone methyltransferase that catalyzes the methylation of H3K36 $[7,8]$, the authors further examined whether SETD2 is responsible for K525 methylation of STAT1. Indeed, SETD2F2 overexpression enhanced the monomethylation of STAT1, whereas silencing of SETD2 reduced the K $525 \mathrm{me} 1$ of STAT1 induced by IFN- $\alpha$ stimulation. An in vitro methylation assay further revealed direct mono-methylation of STAT1 by SETD2. In addition, the authors found that SETD2 selectively catalyzes IFN- $\alpha$-induced trimethylation of H3K36 of some ISG gene loci to promote their expression. Together, these results indicate the dual function of SETD2 in the type I IFN response, revealing a novel regulatory mechanism wherein SETD2 is critically involved in the type I IFN-induced antiviral immune 
responses by targeted methylation of both STAT1 and H3K36 on its target genes.

There are some interesting questions raised by these discoveries. First, as the authors point out, to what extent does SETD2 contribute to the regulation of the broad spectrum of genes targeted by STAT1? Also interesting is whether the SETD2-medited STAT1 activation is additionally required by other cytokine pathways, such as IL-27, which also utilizes STAT1 for its function [9], and whether SETD2 is also involved in the activation of other STAT proteins. In the context of the regulation of oncogenesis, the activity of STAT1 is either oncogenic or anti-oncogenic [10,11], and it may be that SETD2 contributes to the regulation of one, or both of these facets of STAT1 function. Also, given that SETD2 has been shown to have tumor suppressor activity [12], the SETD2-STAT axis discovered by Chen and colleagues may have some implications in the regulation of oncogenesis. In conclusion, the work by Chen and colleagues opens a new chapter in the intricate cytokine-STAT signaling pathways with clinical implications.

Hideyuki Yanai ${ }^{1}$, Tadatsugu Taniguchi ${ }^{1}$

${ }^{1}$ Department of Molecular Immunology, Institute of Industrial Science, The University of Tokyo and Max Planck-The University of Tokyo Center for Integrative Inflammology, Komaba 4-6-1, Meguro-ku, Tokyo 153-8505, Japan Correspondence: Tadatsugu Taniguchi E-mail: tada@m.u-tokyo.ac.jp

\section{References}

1 Velazquez L, Fellous M, Stark GR, et al. Cell 1992; 70:313-322.

2 Schindler C, Shuai K, Prezioso VR, et al. Science 1992; 257:809-813.

3 Harada H, Fujita T, Miyamoto M, et al. Cell 1989; 58:729-739.

4 Tamura T, Yanai H, Savitsky D, et al. Annu Rev Immunol 2008; 26:535-584.

5 Chen K, Liu J, Liu S, et al. Cell 2017; 170:492-506.

6 de Weerd NA, Nguyen T. Immunol Cell Biol 2012; 90:483-491.

7 Edmunds JW, Mahadevan LC, Clayton AL. EMBO J 2008; 27:406-420.

8 Yuan W, Xie J, Long C, et al. J Biol Chem 2009; 284:15701-15707.

9 Amadi-Obi A, Yu CR, Liu X, et al. Nat Med 2007; 13:711-718.

10 Kaplan DH, Shankaran V, Dighe AS, et al. Proc Natl Acad Sci USA 1998; 95:75567561.

11 Yu H, Jove R. Nat Rev Cancer 2004; 4:97105.

12 Li J, Duns G, Westers H, et al. Oncotarget 2016; 7:50719-50734. 